\title{
Analysis of Beta Wave Epileptic EEG Signals Based on Symbolic Transfer Entropy
}

\author{
Xiang Ye, Tian Tian, Tianpei Xu \\ Image Processing and Image Communications Key Lab., \\ Nanjing Univ. of Posts \& Telecomm. \\ Nanjing, China
}

\begin{abstract}
Epilepsy is a common neurological diseases caused by abnormal discharge of neurons in the brain. the attack is sudden and repeated characteristics. Therefore, in order to advance seizure prediction has important meaning for patients to take timely measures in this paper, the seizures in patients with EEG by using the method of symbolic transfer entropy are research and analysis, Through the EEG signal of epilepsy patients during attack and normal human alpha wave is extracted, By using the method of symbolic transfer entropy for analysis and research, Prior to the transfer characteristics which have been analyzed under entropy alpha wave component, this paper starts from beta wave components. then make a study by using the method of symbolic transfer entropy, the study found that using this method can differentiate the normal EEG and EEG in patients with epilepsy, Also found that the existence of nonlinear large amount of time series of EEG. This method is also proved symbolic transfer entropy based algorithm can be used to analyze the EEG signals fully, reveals the difference between epileptic EEG and normal EEG, clinical contribution made certain detection and prediction of epilepsy.
\end{abstract}

Keywords-symbolic transfer entropy; beta wave; epileptic EEG; detection; prediction

\section{INTRODUCTION}

Epilepsy is commonly known as "epilepsy" or "epilepsy". Epilepsy is a kind of consciousness, feeling, nerve function and mental anomaly of plant disease that is caused by brain neurons abnormal discharge of paroxysmal[1-3]. There are many kinds of pathogenic factors. For symbolic transfer entropy algorithm[4]. We intend to firstly achieve a symbolic analysis of sequence, symbolic dynamics is the mathematical theory gradually developed in 20's 20th century. With the in-depth study of chaos, symbolic dynamics gradually become an important method for the analysis of chaos and complex sequence.

The analysis of symbolic time series[4,5] is developed as a new analysis method from the symbolic dynamic theory, chaotic time series and information theory. It can provide a fast, simple and efficient processing method for signal analysis. The symbolic essence is divided the continuous sign into several intervals according to certain rules, transfer the many continuous possible values into several symbolic sequences with discrete values. Although in this process, original sequence is missing some details, but dynamic characteristic is not lost. So it also can capture the large scale information and reduce the effects of dynamic noise and

\author{
Jun Wang \\ Image Processing and Image Communications Key Lab., \\ Nanjing Univ. of Posts \& Telecomm \\ Nanjing, China \\ email: wangj@njupt. edu. cn
}

measurement noise. This process is also called "coarse-grained".

The purpose of this paper is applying the symbolic transfer entropy to the EEG signal for distinguishing epileptic EEG and normal EEG signal, which reveal the difference of epileptic EEG and normal EEG. It makes certain contribution to clinical detection and prediction of epilepsy.

\section{PRINCIPLES}

Dividing the original division of time series data into a series of interval and according to the original dates falling in the interval, it can turn the original data symbols into the corresponding symbols. Thus we can turn the simulated and continuous sequences into symbolic sequences.

The EEG and ECG signal are continuous time series. We usually symbolize the continuous time series $\mathrm{X}$ $=\left\{X_{1}, X_{2}, \ldots, X_{i}, \ldots, x_{N}\right\}$. The length of the $\mathrm{X}$ is $\mathrm{N}$ and the symbolic series is $\mathrm{S}=\left\{S_{1}, S_{2}, \ldots, S_{i}, \ldots, S_{N}\right\}$, $S_{i} \in \mathrm{A},(\mathrm{A}=0,1,2,3)$. In the process of symbolization, although inevitably losting some detail information, the dynamics characteristics of time series is preserved and the numerical calculation of time series is in increasing speed. Symbolic methods used are as follows:

$$
S_{i}\left(x_{i}\right)= \begin{cases}0: & \mu_{1}<x_{i} \leq(1+a) \mu_{1} \text { or }(1+a) \mu_{2} \leq x_{i}<\mu_{2} \\ 1: & (1+a) \mu_{1}<x_{i} \leq \infty \text { or }-\infty \leq x_{i}<(1+a) \mu_{2} \\ 2: & (1-a) \mu_{1}<x_{i} \leq \mu_{1} \text { or } \mu_{2} \leq x_{i}<(1-a) \mu_{2} \\ 3: & (1-a) \mu_{2}<x_{i} \leq(1-a) \mu_{1}\end{cases}
$$

Here, $i=1,2, \cdots \cdots, N, \mu_{1}$ is the mean of $x_{i}$ in $\mathrm{X}$ sequence which is greater than zero, $\mu_{2}$ is the mean of $x_{i}$ in $\mathrm{X}$ sequence which is less than zero and a is Constant parameters, in the process of symbolization. If the a value is improper, The original time series will lose some details, so, we take $a=0.05$.

Transfer entropy is a parameter that quantifying the related degree of two systems or the degree of chaos. It can be used for physiological signal data processing of finite length. 2000

Transfer entropy definition was proposed by Schreiber in 


$$
T_{J->I}=\sum p\left(i_{n+1}, i_{n}^{(k)}, j_{n}^{(l)}\right) \log \frac{p\left(i_{n+1} \mid i_{n}^{(k)}, j_{n}^{(l)}\right)}{p\left(i_{n+1} \mid i_{n}^{(k)}\right)}
$$

$i_{n}, j_{n}$ represent the state at $\mathrm{n}$ times of tow sequences: I sequence, $\mathrm{J}$ sequence. $i_{n}^{(k)}$ represents a string of length $\mathrm{k}$ as $i_{n-k+1}, \cdots \cdots, i_{n}$, By the same token, $j_{n}^{(l)}$ represents a string of length 1 as $j_{n-k+1}, \cdots \cdots, j_{n}$. Usually in order to simplify calculation, we generally take $\mathrm{k}=1, \mathrm{I}=1$.

For symbolic transfer entropy algorithm, the realization of the method is as follows: a time sequence $x(t), t=1,2, \ldots$ then the phase space reconstruction for:

$$
X_{t}=\{x(t), x(t+\tau), \cdots \cdots, x(t+(m-1) \tau)\}
$$

Any arbitrary phase point have $m$ dimensional components, where $\mathrm{m}$ represents embedding dimension, $\tau$ represent delay time. Then we put the $m$ dimensional components of $X_{t}$ in ascending order:

$$
X_{t}=\left\{x\left(t+\left(k_{t 1}-1\right) \tau\right), x\left(t+\left(k_{t 2}-1\right) \tau\right), \cdots \cdots, x\left(t+\left(k_{t m}-1\right) \tau\right)\right\}
$$

It can take the $k_{t 1}, k_{t 2}, \cdots \cdots, k_{t m}$ to construct a new time series, $x_{t}=\left(k_{t 1}, k_{t 2}, \cdots \cdots, k_{t m}\right)$.

In this agreement: if two elements have equal value, $x\left(t+\left(k_{t 1}-1\right) \tau\right)=x\left(t+\left(k_{t 2}-1\right) \tau\right) \quad, \quad$ we take $x\left(t+\left(k_{t 1}-1\right) \tau\right) \leq x\left(t+\left(k_{t 2}-1\right) \tau\right)$ and we think that $X_{t}$ have $m$ ! values.

The same time on the same individuals collected two lead EEG time sequence. Two time sequence number $\left\{x_{i}\right\}$ and $\left\{y_{i}\right\}$, separately for each of the whole sequence may appear in the statistics. The relative frequency and calculate the various situation appears as a probability. Calculating the STE , the calculation process of STE are as follows:

$$
\begin{gathered}
h_{1}=-\sum p\left(x_{t+1}, x_{t}, y_{t}\right) \log _{2} p\left(x_{t+1} \mid x_{t}, y_{t}\right) \\
h_{2}=-\sum p\left(x_{t+1}, x_{t}, y_{t}\right) \log _{2} p\left(x_{t+1} \mid x_{t}\right) \\
p\left(x_{t+1} \mid x_{t}, y_{t}\right)=\frac{p\left(x_{t+1}, x_{t}, y_{t}\right)}{p\left(x_{t}, y_{t}\right)} \\
p\left(x_{t+1} \mid x_{t}\right)=\frac{p\left(x_{t}, x_{t+1}\right)}{p\left(x_{t}\right)}
\end{gathered}
$$

$$
\begin{aligned}
& T_{Y, X}^{S}=h_{2}-h_{1}=\sum p\left(x_{t+1}, x_{t}, y_{t}\right) \log _{2} \frac{p\left(x_{t+1} \mid x_{t}, y_{t}\right)}{p\left(x_{t+1} \mid x_{t}\right)} \\
& =\sum p\left(x_{t+1}, x_{t}, y_{t}\right) \log _{2} \frac{p\left(x_{t+1}, x_{t}, y_{t}\right) p\left(x_{t}\right)}{p\left(x_{t}, y_{t}\right) p\left(x_{t}, x_{t+1}\right)}
\end{aligned}
$$

In the formula, $h_{1}$ express $y_{t}$ and $x_{t+1}$ are not independent by $x_{t}, y_{t}$ to test the entropy of next sampling point $x_{t+1}$. The formula $h_{2}$ expresses $y_{t}$ and $x_{t+1}$ is independent by ${ }_{t}$ to test the entropy of next sampling point which describing the degree of correlation and randomness between two time sequence quantitatively.

\section{DATA PROCESSING AND ANALYSIS}

\section{A. Experimental Data}

In this paper, by the use of General Hospital of Nanjing Military Region collected from clinical diagnosis information,take the EEG signal from epilepsy patients and normal people in the database, epilepsy patients and normal subjects were divided into two groups, each group had ten objects, The EEG data for each object includes 24 lead, The record length is greater than 1 minutes, The sampling period is $1024 \mathrm{~Hz}$, Among two groups are both of male and female.We study the transfer entropy of two column lead date:Fp1 and Fp2(experiment proof, the symbolic transfer entropy of Fp1andFp2 have good discrimination. so, take Fp1,Fp2 lead as the object of study). In the following, we record epilepsy group "epilepsy", normal group for "normal".

\section{B. Experimental Procedure}

1) Extraction of alpha wave. The normal EEG and epileptic EEG difference is not very obvious.Before we will have for the alpha wave components to do some analysis, we start from the beta wave components.we should process the date firstly, extract the beta wave epileptic EEG and normal EEG ,then calculate the transfer entropy of the beta wave, Observe whether can effectively distinguish epileptic EEG and normal EEG, time sequence diagrams of lead Fp1. After filtering algorithm, the extracted beta wave, In order to test the effect of filtering, then turn the time domain of beta wave into frequency domain. we find beta wave extracted regularly, and filtering algorithm is more reasonable, Epileptic EEG frequency diagram of beta wave in lead Fp1, the single mainly concentrated in the $14 \sim 30 \mathrm{~Hz}$, meeting the requirements.

2) Analysis of different length of the transfer entropy. In order to verify through time series symbolization treatment, computing the transfer entropy whether has the advantage of speed, a time series we each take four epileptic EEG and normal EEG signal from the database,calculating the transfer entropy between two lead Fp1andFp2 in different length.In the experiment,we take 1000、2000、3000 ... 8000 points,to calculate the transfer entropy as shown in Figure 2.1. 

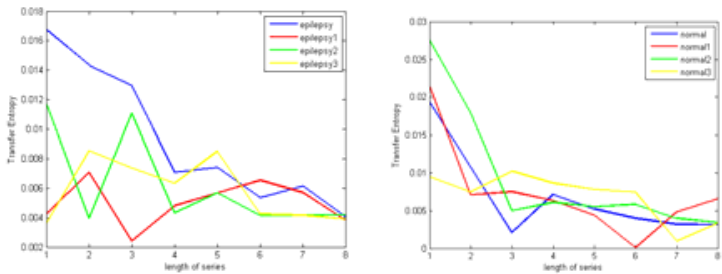

FIGURE I. THE EPILEPTIC EEG ENTROPY TRANSFER VALUE IN DIFFERENT LENGTHS

The results showed that, the value of transfer entropy in each sequence is almost convergent .after reaching a certain length., thousands of points can get consistent results, so considering the epileptic EEG and normal EEG pattern, we go to select the $L=4000$ as the selection of length, This symbolic transfer entropy method not only needs less data, and with high computational efficiency.

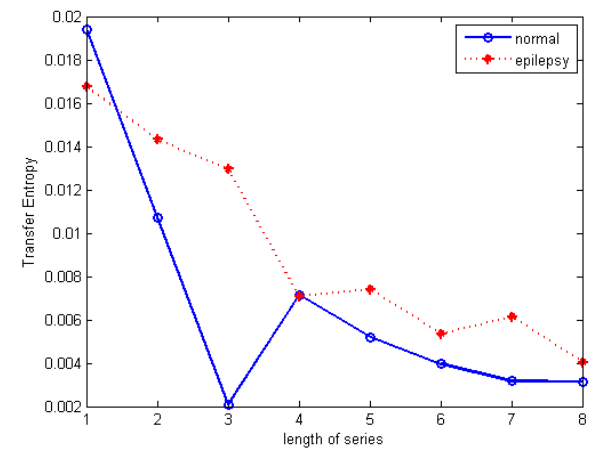

FIGURE II. NORMAL AND EPILEPSY TRANSFER ENTROPY VALUES

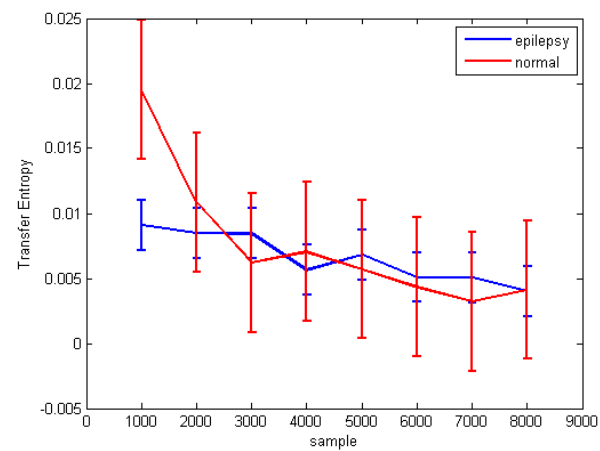

FIGURE III. TRANSFER ENTROPY ERROR BARS IN FIG.

When putting together epileptic EEG and normal EEG in Figure 2.2,we found that discrimination is not great.Besides, we have to transfer entropy four normal EEG and epileptic EEG as error bars which correspond to the length of the chart in Figure 2.3, nor does it have a good discrimination, so we consider the transfer entropy change at different times.

3) Analysis of different time of the transfer entropy. The heart is a very complex system, In addition to unipolar and bipolar effects of heart, many factors in the process will change with the change of time,the coupling effect between channels will also changes over time, So we need to Analysis temporal properties of transfer entropy on the time.

For epileptic EEG and normal EEG, we are beginning to take a length of 4000 data from the initial moment, To calculate the transfer entropy, then every take a length of 4000 data start to the 1000 point, calculation of transfer entropy, obtains two kind of signal transfer entropy change with time is shown in Fig. 2.4.
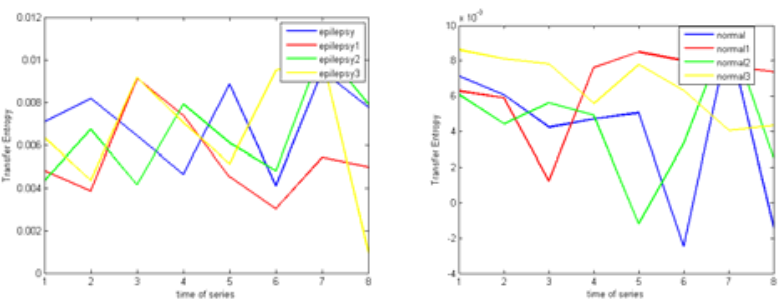

FIGURE IV.DIFFERENT PERIODS THE TRANSFER ENTROPY VALUE OF EPILEPTIC EEG

From the above it can be seen from the graph, two kinds of signal transfer entropy are changing with the change of time, the paper explains two kinds of signal after lead system transmission, coupling effect between lead Fp1 and lead Fp2 are changing with the time change.

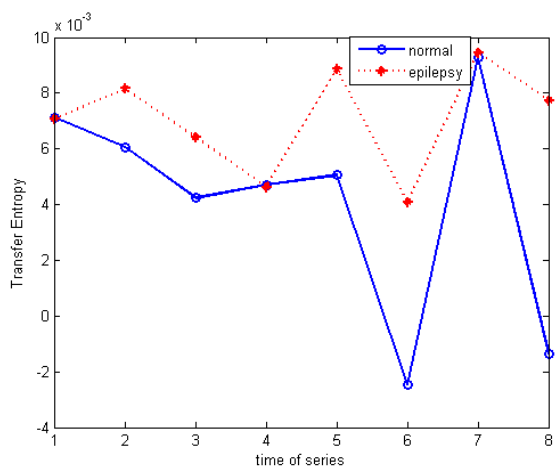

FIGURE V. NORMAL AND EPILEPSY TRANSFER ENTROPY VALUES

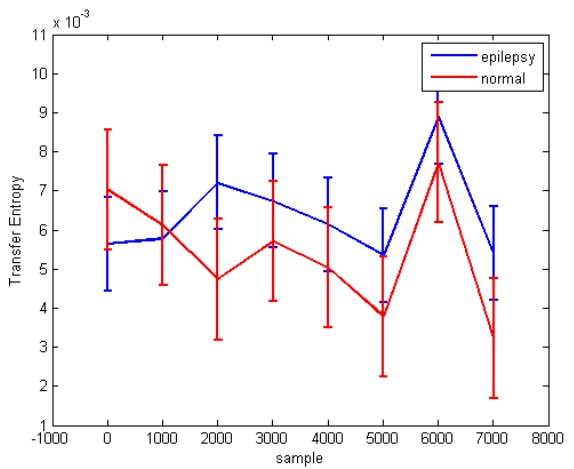

FIGURE VI.TRANSFER ENTROPY ERROR BARS IN FIG.

Besides, it can be seen from Figure 2.5, the coupling effect between the two signal lead Fp1 and lead Fp2 have changed over time , but we found that the transfer entropy of epileptic EEG changes over time will not be negative. transfer entropy of normal EEG changes occur over time, negative, indicating 
epileptic EEG coupling sometimes change direction. From Figure 2.6, we can see that the transfer entropy of normal EEG and epileptic EEG have been a clear distinction over time, the value of the transfer entropy of epileptic EEG changes over time is greater than the normal EEG transfer entropy, and the transfer entropy of epileptic EEG absolute difference is greater than the normal EEG changes transferred entropy.

4) Analysis of significant differences between the transfer entropy. Finally, In order to verify whether a significant difference between the second and third step experiment calculated signal transfer entropy, we have them independent samples t test.

Using SPSS software for two independent samples t test signal.

For epileptic EEG and normal EEG, when compared to the length of the analysis, SPSS software was used to calculate the second stage of the experiment $t=0.939$, look-up table $t=0.939>t_{0.05(8)}=1.8595(p<0.05$ the difference was statistically significant), So consider the length of the analysis and comparison of methods can not clearly distinguish epileptic EEG and normal EEG.

For epileptic EEG and normal EEG, when compared to the time of the analysis, SPSS software was used to calculate the third stage of the experiment $t=1.881$, look-up table $t=1.881>t_{0.05(8)}=1.8595$, So consider the time of the analysis and comparison of methods can also clearly distinguish epileptic EEG and normal EEG.

\section{CONCLUSIONS}

Epilepsy is a brain neurons caused by excessive discharge of brain dysfunction, the onset of brain damage is irreversible, and therefore seriously endanger human health. In this paper, the use of symbolic transfer entropy method EEG epileptic seizures during the study and analysis. By EEG normal EEG and epilepsy seizures when taken out of the beta wave, Then the data of different length and time, using the method of symbolic transfer entropy research and analysis. Transfer entropy was subjected to independent samples t test. Finally, we found that the method can effectively distinguish between normal EEG and epileptic EEG and also found that there are a lot of non-linear components of the EEG time series. Fully proved symbolic transfer entropy-based algorithm can be used to analyze EEG. That revealed the difference between epilepsy EEG and normal EEG. We can make some clinical contribution for epilepsy detection and prediction.

\section{ACKNOWLEDGEMENTS}

In this paper, the project supported by the National Natural Science Foundation of China (Grant Nos. 61271082,61201029,61102094,61401518), the Natural Science Foundation of Jiangsu Province (Grant Nos.BK20141432) , the Foundation of Nanjing General Hospital of Nanjing Military Command (2014019) and the Fundamental Research Funds for the Central Universities (FY2014LX0039).

\section{REFERENCES}

[1] Meng Q F, zhou W, Chen Y H, Peng Y H 2010 Acta Phys. Sin. 59 123-130.

[2] Ma Q L, Bian C H, Wang J 2010 Acta Phys. Sin. 59 4480-4484

[3] Wang J, Ma Q-L 2008 Chin. Phys. B 174424

[4] Staniek M, Lehnertz K 2007 Phys. Rev. Lett. 99204101

[5] Shen W, Wang J 2010 Acta Phys. Sin. 60118702. 